\title{
Acid-mediated tumor invasion as a function of nutrient source location
}

\author{
Silvia A. Menchón (1) \\ IFEG-CONICET and FaMAF-Universidad Nacional de Córdoba, Ciudad Universitaria, Córdoba X5016LAE, Argentina
}

(Received 26 April 2019; published 29 August 2019)

\begin{abstract}
Cancer cells have an altered metabolism that increases acid production driving to an extracellular $p \mathrm{H}$ significantly lower than normal. This leads to normal cell death, and extracellular matrix degradation allowing the formation of an interstitial gap between cancer and healthy cells. In this work, we present a mathematical model to study the interstitial gap formation and evolution considering a tissue with a non-uniform nutrient distribution. Our results indicate that the interstitial gap onsets at the region with highest nutrient consumption. Due to the gap formation, cancer cells near the interface have more nutrient and space availability. This induces cancer cell reproduction and migration toward the nutrient source. Our simulations suggest a strong correlation between gap size and the distance to the nutrient source. Although we do not find a correlation between tumor growth speed and gap size, our results indicate a high risk of metastasis for tumors that develop an interstitial gap, emphasizing the importance of gap detection as a hallmark for cancer invasion.
\end{abstract}

DOI: 10.1103/PhysRevE.100.022417

\section{INTRODUCTION}

It is well known that cancer cells have an altered metabolism. Warburg was the first who observed that cancer cells use the glycolytic pathway, rather than oxidative phosphorylation, despite sufficient oxygen supply [1]. This phenomenon is known as the "Warburg effect". Although anaerobic glycolysis is very inefficient, since the adenosine triphosphate (ATP) production per molecule of glucose is significantly lower compared to the normal oxidation pathway [2], the acid-mediated tumor invasion hypothesis considers the Warburg effect as a cancer cell advantage. This is because the Warburg effect increases acid production in tumor cells driving to an extracellular $p \mathrm{H}$ significantly lower than normal [3]. This leads to normal cell death, and extracellular matrix degradation, that would enhance cancer cell migration and invasion capabilities.

Gatenby and Gawlinski were the first who studied the acidmediated invasion hypothesis considering a reaction-diffusion differential equation system [4]. They found that an interstitial gap between cancer and healthy cells is established for aggressive tumors. This initial model was the starting point for more generalized models considering, for instance, the early stages of cancer growth [5], cooperative interaction between tumor and stromal cells [6], and acid-mediated tumor cell death [7]. In general, the presence of a gap at the tumor-host interface is related with aggressive tumors and this gap is a consequence of high acidity levels. However, Ref. [6], states that, in some cases, increasing tumor acidity may prevent tumor invasion. To understand how this tumor-host interface is related with tumor progression and the lactate production has a huge diagnostic potential.

In general, most of the mathematical models consider homogeneous environment or well-mixed cells [8]. However,

\footnotetext{
*silvia.menchon@unc.edu.ar
}

the effect of inhomogeneities may change tumor shapes and invasion capabilities [8-11]. In this work, we generalized our previous model [12] including new rules to introduce acid production. One of the key characteristics of our approach is the non-uniform nutrient distribution, since we consider only one blood vessel at the bottom of the network. Our results suggest that an interstitial gap is generated along the tumor-host interface and its size is strongly correlated with the distance to the nutrient source. Although we do not find a strong correlation between gap size and speed of growing, the presence of a gap suggest highly aggressive tumors in terms of larger metastatic spread probabilities, because there is a clear privilege direction of growing toward the blood vessel, even if there is enough free space in other regions of the tumor.

\section{THE MODEL}

We consider the cancer growth model presented in Refs. [12,13] for describing avascular tumor growth. In this model the tissue is represented by a network in which each point is associated with a volume element that contains many cells, nutrient molecules and excess of $\mathrm{H}^{+}$ions. Healthy, cancerous, and dead cells coexist at each node point, their concentrations being denoted by $h(\vec{i}, t), c(\vec{i}, t)$, and $d(\vec{i}, t)$, respectively. The extra-cellular matrix degradation is represented by the presence of cell free space, which we denote by $e(\vec{i}, t)$; since for each node, the total concentration is considered to be uniform and normalized, the normalization equation is generalized to $h(\vec{i}, t)+c(\vec{i}, t)+d(\vec{i}, t)+e(\vec{i}, t)=$ 1. Cancer cells produce an excess of $\mathrm{H}^{+}$ions while they consume nutrients due to their aerobic glycolytic metabolism. The acid diffuses in the tissue with diffusion coefficient $\alpha_{W}$ and has a constant rate of degradation, $d_{W}$. The excess $\mathrm{H}^{+}$ ion concentration at the $\vec{i}$-node is denoted by $W(\vec{i}, t)$. For simplicity, we consider a single critical nutrient which diffuses through the tissue, with diffusion coefficient $\alpha^{\prime}$. We call it free 
nutrient and denote its concentration at the $\vec{i}$-node by $p(\vec{i}, t)$. Free nutrient is absorbed by the healthy cells at rate $\gamma_{0}$.

The rules governing cancer growth are as follows:

R1. Feeding. Free nutrient is absorbed by cancer cells and converted into bound nutrient. The absorption rate is proportional to $p(\vec{i}, t)$ at low free nutrient concentrations and it saturates to a constant value, $\gamma_{a s}$, at high concentrations (see Ref. [14]). We model the absorption rate by

$$
\gamma(\vec{i}, t)=\gamma_{a s}\left(1-e^{-p(\vec{i}, t)}\right) .
$$

This rule only modifies free and bound nutrient concentrations as follows:

$$
\begin{aligned}
& p(\vec{i}, t) \rightarrow p(\vec{i}, t)-\tau \gamma(\vec{i}, t) c(\vec{i}, t), \\
& q(\vec{i}, t) \rightarrow q(\vec{i}, t)+\tau \gamma(\vec{i}, t) c(\vec{i}, t),
\end{aligned}
$$

where $q(\vec{i}, t)$ is the bound nutrient concentration and $\tau$ the time step.

$\mathrm{R} 2$. Consumption. Bound nutrient is consumed by $\vec{i}$-node cells at the rate

$$
\beta(\vec{i}, t)=\beta_{a s}\left(1-e^{-q(\vec{i}, t) / c(\vec{i}, t)}\right),
$$

where the denominator $c(\vec{i}, t)$ has been included in the exponent because each cell can consume only its own bound nutrient. In order to model the effect of an aerobic glycolytic metabolism, an excess of $\mathrm{H}^{+}$ions is produced with rate $\gamma_{W} \beta(\vec{i}, t)$, when cancer cells at the $\vec{i}$-node consume nutrients. Thus

$$
\begin{aligned}
q(\vec{i}, t) & \rightarrow q(\vec{i}, t)-\tau \beta(\vec{i}, t) c(\vec{i}, t), \\
W(\vec{i}, t) & \rightarrow W(\vec{i}, t)+\tau \gamma_{W} \beta(\vec{i}, t) c(\vec{i}, t) .
\end{aligned}
$$

R3. Cancer cell death. If the average amount of bound nutrient per cell, $q(\vec{i}, t) / c(\vec{i}, t)$, is below a given threshold $Q_{D}$, a fraction $r_{D} c(\vec{i}, t)$ of cancer cell dies. Cancer and dead cell populations are modified by

$$
\begin{aligned}
& c(\vec{i}, t) \rightarrow c(\vec{i}, t)\left(1-r_{D} \Theta\left(c(\vec{i}, t) Q_{D}-q(\vec{i}, t)\right)\right), \\
& d(\vec{i}, t) \rightarrow d(\vec{i}, t)+r_{D} c(\vec{i}, t) \Theta\left(c(\vec{i}, t) Q_{D}-q(\vec{i}, t)\right),
\end{aligned}
$$

where $\Theta(x)$ is the Heaviside's step function and $r_{D}$ is a constant.

R4. Healthy cell death. If the excess $\mathrm{H}^{+}$ion concentration is above a given threshold $Q_{W}$, a fraction $r_{W} h(\vec{i}, t)$ of healthy cells dies. We assume that they die by activating their apoptotic pathways; thus, they can be absorbed, [15], and cell free space is generated by increasing $e(\vec{i}, t)$. The corresponding equations are

$$
\begin{aligned}
& h(\vec{i}, t) \rightarrow h(\vec{i}, t)\left(1-r_{W} \Theta\left(W(\vec{i}, t)-Q_{W}\right)\right), \\
& e(\vec{i}, t) \rightarrow e(\vec{i}, t)+r_{W} h(\vec{i}, t) \Theta\left(W(\vec{i}, t)-Q_{W}\right),
\end{aligned}
$$

where $r_{W}$ is a constant.

R5. Mitosis. If the average amount of bound nutrient per cell is above a given threshold $Q_{M}\left(Q_{M}>Q_{D}\right)$, the concentration of cancer cells may increase up to $\left.r_{M} c \vec{i}, t\right)$ depending on the space availability, where $r_{M}$ is a constant. If $e(\vec{i}, t)=0$ we should recover the Refs. $[12,13]$ mitosis rule. If $e(\vec{i}, t) \neq 0$, new cancer cells fill the available free space before replacing healthy cells. Thus, when mitosis rule has to be applied at the $\vec{i}$-node, cell free space is reduced by a fraction

$$
g(\vec{i}, t)=e(\vec{i}, t)+\left(r_{M} c(\vec{i}, t)-e(\vec{i}, t)\right) \Theta\left(e(\vec{i}, t)-r_{M} c(\vec{i}, t)\right) .
$$

If $e(\vec{i}, t)<r_{M} c(\vec{i}, t)$, cancer cell concentration may still increase up to $r_{M} c(\vec{i}, t)-e(\vec{i}, t)$ by replacing healthy cells. Thus, a fraction,

$$
\begin{aligned}
f(\vec{i}, t)= & h(\vec{i}, t)-h(\vec{i}, t) \Theta\left(e(\vec{i}, t)-r_{M} c(\vec{i}, t)\right) \\
& +\left(r_{M} c(\vec{i}, t)-e(\vec{i}, t)\right) \Theta\left(h(\vec{i}, t)-\left(r_{M} c(\vec{i}, t)\right.\right. \\
& -e(\vec{i}, t))) \Theta\left(r_{M} c(\vec{i}, t)-e(\vec{i}, t)\right),
\end{aligned}
$$

of healthy cells is transformed into cancer cells. If $e(\vec{i}, t) \geqslant$ $r_{M} c(\vec{i}, t)$ the last term in the above equation is zero and $f(\vec{i}, t)=0$; it means that the cell free space available at node $\vec{i}$ has been enough to contain all the new cancer cells. If $e(\vec{i}, t)<r_{M} c(\vec{i}, t)$, the second term in the $f(\vec{i}, t)$ expression is zero and $f(\vec{i}, t)$ takes the minimal value between $h(\vec{i}, t)$ and $r_{M} c(\vec{i}, t)-e(\vec{i}, t)$. The equations after applying mitosis rule are as follows:

$$
\begin{aligned}
& c(\vec{i}, t) \rightarrow c(\vec{i}, t)+(g(\vec{i}, t)+f(\vec{i}, t)) \Theta\left(q(\vec{i}, t)-c(\vec{i}, t) Q_{M}\right), \\
& e(\vec{i}, t) \rightarrow e(\vec{i}, t)-g(\vec{i}, t) \Theta\left(q(\vec{i}, t)-c(\vec{i}, t) Q_{M}\right), \\
& h(\vec{i}, t) \rightarrow h(\vec{i}, t)-f(\vec{i}, t) \Theta\left(q(\vec{i}, t)-c(\vec{i}, t) Q_{M}\right) .
\end{aligned}
$$

R6. Migration. If the average amount of free nutrient per cell, $p(\vec{i}, t) / c(\vec{i}, t)$, is below a migration threshold, $P_{D}$, cancer cells at the $\vec{i}$-node migrate to its neighbor nodes. If there is cell free space availability at the destination node, this can be filled by cancer cells. However, we still assume that healthy cells may be eliminated when cancer cells arrive, since healthy cells are less mobile and aggressive than cancer cells, in such a way that the total cell concentration is preserved, as in Refs. [12,13]. In other words, if there is no enough free nutrient, cancer cells at the $\vec{i}$-node migrate to its neighbor nodes with migration coefficient $\alpha$ if either cell free space or healthy cells are present at the destination node. The corresponding equations for this rule are

$$
\begin{aligned}
c(\vec{i}, t) \rightarrow & c(\vec{i}, t)+\frac{\tau}{\ell^{2}}\left[(h(\vec{i}, t)+e(\vec{i}, t)) \sum_{\vec{i}^{\prime}} \tilde{\alpha}\left(\vec{i}^{\prime}, t\right) c\left(\vec{i}^{\prime}, t\right)\right. \\
& \left.-\tilde{\alpha}(\vec{i}, t) c(\vec{i}, t) \sum_{\vec{i}^{\prime}}\left(h\left(\vec{i}^{\prime}, t\right)+e\left(\vec{i}^{\prime}, t\right)\right)\right], \\
e(\vec{i}, t) \rightarrow & e(\vec{i}, t)+\frac{\tau}{\ell^{2}}\left[\tilde{\alpha}(\vec{i}, t) c(\vec{i}, t) \sum_{\vec{i}^{\prime}} e\left(\vec{i}^{\prime}, t\right)\right. \\
& \left.-e(\vec{i}, t) \sum_{\vec{i}^{\prime}} \tilde{\alpha}\left(\overrightarrow{i^{\prime}}, t\right) c\left(\vec{i}^{\prime}, t\right)\right]
\end{aligned}
$$

where $\tilde{\alpha}(\vec{i}, t)=\alpha \Theta\left(P_{D} c(\vec{i}, t)-p(\vec{i}, t)\right), \ell$ is the spatial discretization, and healthy cell concentration may be computed from the conservation equation. Cancer cell migration depends not only on the local diffusivity but also on the state of the target site. 
R7. Mobility. Since the presence of cell free space reduces the pressure, we assume that a fraction of cancer cells may invade the cell free space at their neighbor nodes. Thus, a node with cell free space could be filled out with cancer cells with a probability $p_{f}$ by choosing a random number of cancer cells, $r_{c}$, from a neighbor node, that is also chosen randomly. The random number $r_{c}$ is a number between 0 and $\min \left(e(\vec{i}, t), c\left(\vec{i}^{\prime}, t\right)\right)$, where $\vec{i}$ - and $\vec{i}^{\prime}$-nodes are neighbor nodes. The parameter $p_{f}$ is also related with cancer invasion, in the sense that the greater $p_{f}$ the more invasive the tumor. Thus

$$
\begin{aligned}
e(\vec{i}, t) & \rightarrow e(\vec{i}, t)-r_{c} \Theta\left(r-p_{f}\right), \\
e\left(\vec{i}^{\prime}, t\right) & \rightarrow e\left(\vec{i}^{\prime}, t\right)+r_{c} \Theta\left(r-p_{f}\right), \\
c(\vec{i}, t) & \rightarrow c(\vec{i}, t)+r_{c} \Theta\left(r-p_{f}\right), \\
c\left(\vec{i}^{\prime}, t\right) & \rightarrow c\left(\vec{i}^{\prime}, t\right)-r_{c} \Theta\left(r-p_{f}\right),
\end{aligned}
$$

where $r$ is a random number between zero and one and the $\vec{i}^{\prime}$-node is only one of the $\vec{i}$-node nearest neighbors, that has been chosen randomly. Rule R7 differs from rule R6 because the last considers cancer cell migration due to a low nutrient concentration, while rule R7 only takes into account local space availability.

As we show, implementation of these rules generates a set of nonlinear difference equations. Iteration equations for the evolution of free nutrient and excess $H^{+}$ion concentrations are given by

$$
\begin{gathered}
p(\vec{i}, t+\tau) \rightarrow p(\vec{i}, t)+\tau\left[\sum_{\vec{i}^{\prime}} \frac{\alpha^{\prime}}{\ell^{2}}\left(p\left(\vec{i}^{\prime}, t\right)-p(\vec{i}, t)\right)\right. \\
\left.\quad-\gamma_{0} p(\vec{i}, t) h(\vec{i}, t)-\gamma(\vec{i}, t) c(\vec{i}, t)\right], \\
\begin{aligned}
W\left(\vec{i}^{\prime}, t+\tau\right) \rightarrow W\left(\vec{i}^{\prime}, t\right)+\tau\left[\sum_{\vec{i}^{\prime}} \frac{\alpha_{W}}{\ell^{2}}\left(W\left(\vec{i}^{\prime}, t\right)-W(\vec{i}, t)\right)\right. \\
\left.+\gamma_{W} \beta(\vec{i}, t) c(\vec{i}, t)-d_{W} W(\vec{i}, t)\right],
\end{aligned}
\end{gathered}
$$

respectively.

In the next section we detail initial and boundary conditions, as well as, the implementation of this model. In this work, we consider an inhomogeneous distribution of free nutrient. This allows us to analyze cancer cell behavior with different local scenarios and since excess $\mathrm{H}^{+}$ion concentration is correlated with the consumption of cancer cells, its production would also be affected by local conditions.

\section{RESULTS}

In this work we represent the tissue of interest by a twodimensional grid $(N \times M)$, with lattice constant $\ell$ and node points $\vec{i}=(i \ell ; j \ell)$, with $i=0,1, \ldots, N$ and $j=0,1, \ldots, M$. The nutrient is supplied by a single capillary vessel situated at the lower edge of the lattice. The nutrient concentration in the blood vessel is constant, $p((i \ell ; 0), t)=P_{0}$, with $i=$ $0,1, \ldots, N$. Periodic boundary conditions are used for the
TABLE I. Numerical values of computational parameters.

\begin{tabular}{lccc}
\hline \hline Symbol & Unit & Value & References \\
\hline$\ell$ & $\mu \mathrm{m}$ & 33 & \\
$\tau$ & $\mathrm{h}$ & 0.001 & \\
$P_{0}$ & $\mathrm{Mm}$ & 5.5 & {$[19]$} \\
$\alpha^{\prime}$ & $\mathrm{cm}^{2} / \mathrm{h}$ & 0.001 & {$[16,20]$} \\
$\gamma$ & $1 / \mathrm{h}$ & 0.002 & {$[16,21]$} \\
$\gamma_{a s}$ & $1 / \mathrm{h}$ & 200 & {$[21,22]$} \\
$\beta_{a s}$ & $1 / \mathrm{h}$ & 5 & {$[23]$} \\
$\alpha$ & $\mathrm{cm}^{2} / \mathrm{h}$ & $8.3 \times 10^{-8}$ & {$[24,25]$} \\
$\alpha_{W}$ & $\mathrm{~cm}^{2} / \mathrm{s}$ & $5 \times 10^{-6}$ & {$[17]$} \\
$d_{W}$ & $1 / \mathrm{s}$ & $10^{-4}$ & {$[4]$} \\
$\gamma_{W}$ & & 0.7 & Estimated \\
\hline \hline
\end{tabular}

left and right boundaries, and absorbing conditions for the upper boundary. Absorbing conditions are also considered for cancer cell and excess $\mathrm{H}^{+}$ion concentrations at the lower boundary, due to the presence of the blood vessel. Initially we consider a healthy tissue with stationary nutrient distribution. At $t=0$ a cancer seed is placed at the center of the lattice, and the tumor evolution starts.

At $t=0$ a tumor starts growing at the center of a completely healthy tissue. On the basis of our previous work [12] the temporal discretization was chosen to be $\tau=0.001 \mathrm{~h}$. Cell growth and division are regulated by the cell cycle. The cellcycle duration is approximately $12 \mathrm{~h}$ in exponentially growing monolayer cultures [16]. Therefore, we inspect the threshold $Q_{M}$ every 12000 steps. We also implement rules R3-R7 every 12000 steps.

Our two-dimensional grid represents a slab of tissue of size $2 \mathrm{~cm} \times 1 \mathrm{~cm}$, and we take $N=600$ and $M=300$. The values for most of the parameters that are related with nutrient diffusion, cancer and healthy cell consumption, cancer cell death, mitosis, and migration, were already discussed in Ref. [12]. Based on Refs. [4,17], the parameter values corresponding to $\alpha_{W}$ and $d_{W}$ were taken as $5 \times 10^{-6} \mathrm{~cm}^{2} / \mathrm{s}$ and $10^{-4} / \mathrm{s}$, respectively. The parameter $\gamma_{W}$ was estimated in order to obtain concentration of $W$ similar to those reported in Ref. [18]. Although Table I summarizes the reference values of the parameters used in most of the simulations, we have also explored the effects of variations in some of them. Most of the parameters related with cancer growth without considering acid production were fixed, and we focus on the effects of changing the parameters related with cancer cell migration as well as acid diffusion and production. In particular, $\alpha, \alpha_{W}$, $\gamma_{W}$, and $p_{f}$ are specified for each figure.

Figure 1 shows snapshots of growing tumors, including live and necrotic cells, 90 days after seeding for $p_{f}=0.5, \alpha=$ $8.3 \times 10^{-8} \mathrm{~cm}^{2} / \mathrm{h}$, and (a) $\alpha_{W}=5 \times 10^{-6} \mathrm{~cm}^{2} / \mathrm{s}, \gamma_{W}=$ 0.7 ; (b) $\alpha_{W}=5 \times 10^{-6} \mathrm{~cm}^{2} / \mathrm{s}, \gamma_{W}=0.9$; and (c) $\alpha_{W}=2 \times$ $10^{-6} \mathrm{~cm}^{2} / \mathrm{s}, \gamma_{W}=0.7$. At a given time, the gap between cancer and healthy cells will be formed by all the nodes for which neither healthy nor cancer cells are present; those nodes are represented by black points in panels (d), (e), and (f). In other words, each of the lower panels of Fig. 1 show the gap between cancer and healthy cells for the corresponding upper panel. If we fix all the parameters and only increase 

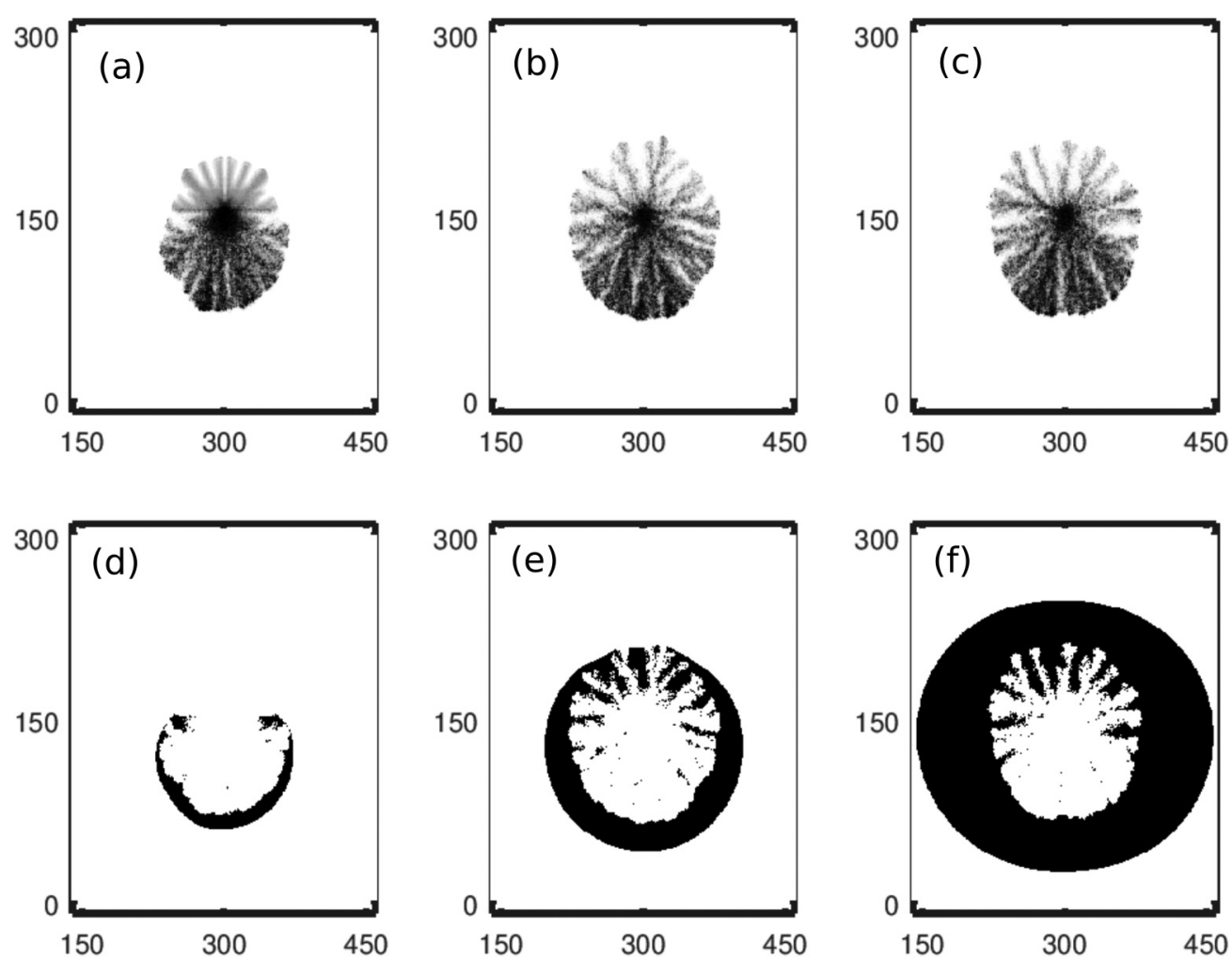

FIG. 1. Snapshots of growing tumors and interstitial gap, 90 days after seeding for $p_{f}=0.5, \alpha=8.3 \times 10^{-8} \mathrm{~cm}^{2} / \mathrm{h}$, and $\alpha_{W}=5 \times$ $10^{-6} \mathrm{~cm}^{2} / \mathrm{s}, \gamma_{W}=0.7$, (a) and (d); $\alpha_{W}=5 \times 10^{-6} \mathrm{~cm}^{2} / \mathrm{s}, \gamma_{W}=0.9$, (b) and (e); $\alpha_{W}=2 \times 10^{-6} \mathrm{~cm}^{2} / \mathrm{s}, \gamma_{W}=0.7$, (c) and (f). Scale color: for upper panels, white color corresponds to $c(\vec{i})+d(\vec{i})=0$ and black color corresponds to $c(\vec{i})+d(\vec{i})=1$; for lower panels, black color corresponds to $c(\vec{i})=d(\vec{i})=h(\vec{i})=0$.

$\gamma_{W}$, the tumor produces more acid, thus healthy cells are more affected. On the other hand, if all parameters are fixed, but $\alpha_{W}$ decreases; acid diffusion is slower. Thus, there is a local increasing in the acid concentration. In this sense, simulations with a larger $\gamma_{W}$ or smaller $\alpha_{W}$ have the same effect: in both cases the local concentration of $W$ increases. Due to the asymmetric distribution of nutrient, there is more local nutrient availability on the lower half of the tissue; and thus more local $W$ production. This is the reason why the gap starts at the bottom of the tumor. Depending on $W$ production and diffusion the gap could surround all the tumor, but still, in general, it does not show an uniform width. In this model, nutrient and space competitions play an important role; when healthy cells are killed, there is more space and nutrient availability. In particular, cancer cells near the gap can consume and migrate more than those in the same conditions, but without considering acid production. Our simulations indicates that tumors clearly show a privilege direction of growing toward the blood vessel, even if the gap is present around all the tumor boundary. In order to analyze this, we define the mean tumor radius as $R=\langle r\rangle=(1 / N) \sum_{i=1}^{N} r_{i}$, where the sum is over the $N$ nodes at the tumor edge and $r_{i}$ is the distance from node point $\vec{i}$ to tumor center of mass. We also define the minimal (maximal) distance from the tumor to the vessel as $j_{\min } \ell$ $\left(j_{\max } \ell\right)$, where $j_{\min }\left(j_{\max }\right)$ is such that $c\left(\left(i \ell ; j_{\min } \ell\right), t\right) \neq 0$ $\left[c\left(\left(i \ell ; j_{\max } \ell\right), t\right) \neq 0\right]$ for some $i$, and $c((i \ell ; j \ell), t)=0$ for all $i$ and $j<j_{\min }\left(j>j_{\max }\right)$. With the same procedure, $i_{\min }$ and $i_{\max }$ are also defined. In order to compare results with and without acid production, the sub-index zero will be used to identify realizations without considering acid production. Figure 2(a) shows the ratio between $R$ and $R_{0}$ versus time for the realizations that have been shown in Fig. 1(a) dotted
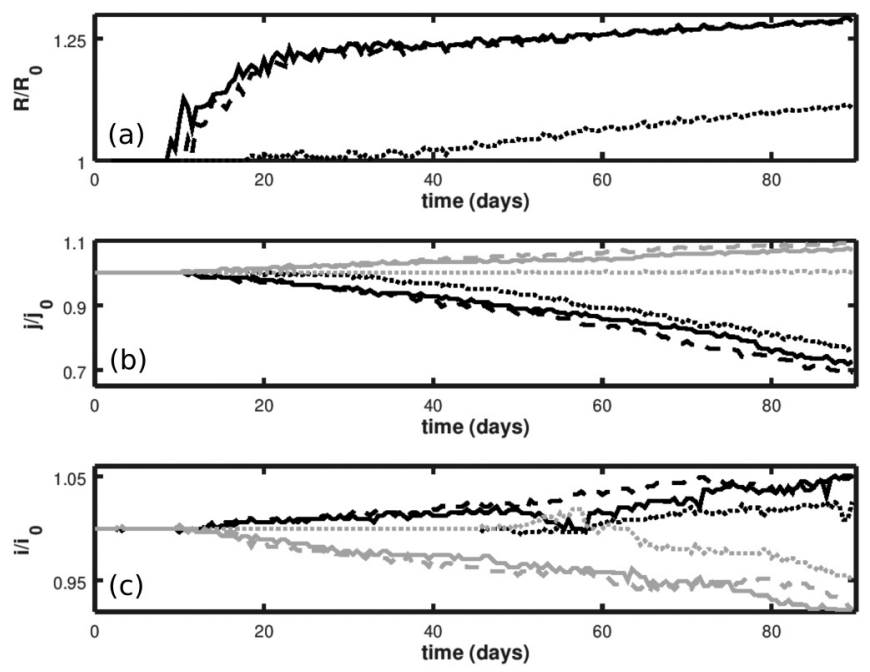

FIG. 2. Ratio between tumor radii with and without considering lactate production, (a). Ratio between minimal (black), and maximal (gray), distance to the blood vessel with and without considering lactate production (b). Ratio between $i_{\min }$ (black) and $i_{\max }$ (gray), with and without considering lactate production (c). For all plots, dotted, dashed, and solid lines correspond to realizations that have been shown in Fig. 1(a), (b), and (c), respectively. 


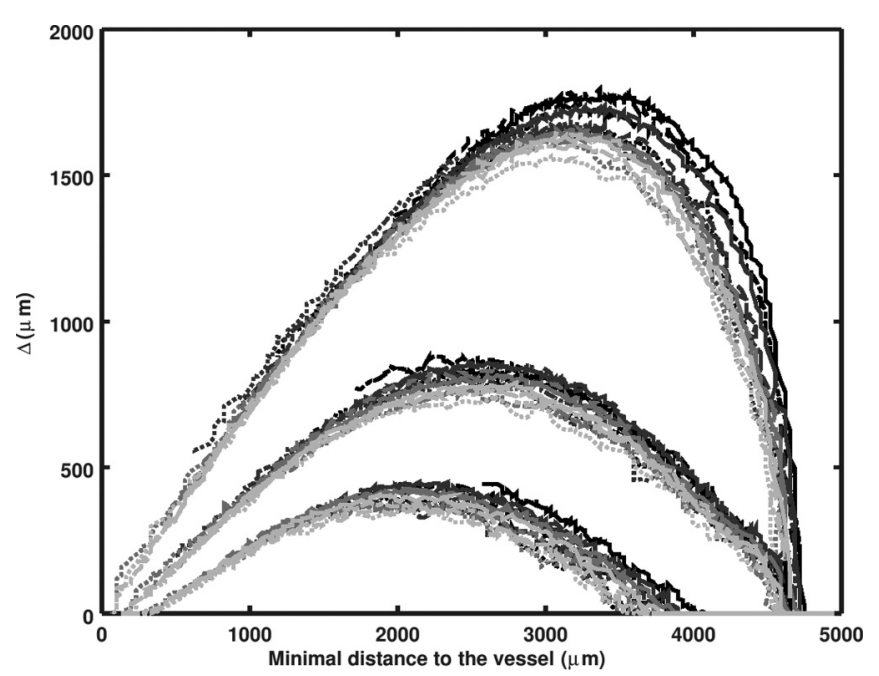

FIG. 3. Interstitial gap size versus the minimal distance from the tumor to the blood vessel. Realizations are associated with three groups, the first one has the lowest maximum and it corresponds to $\alpha_{W}=5 \times 10^{-6} \mathrm{~cm}^{2} / \mathrm{s}, \gamma_{W}=0.7$; the curves in the middle correspond to $\alpha_{W}=5 \times 10^{-6} \mathrm{~cm}^{2} / \mathrm{s}, \gamma_{W}=0.9$; and the third group, corresponding to the highest maximum, includes realizations with $\alpha_{W}=2 \times 10^{-6} \mathrm{~cm}^{2} / \mathrm{s}, \gamma_{W}=0.7$. For all plots, the following $p_{f}$ values has been considered: 0.25 (solid lines); 0.5 (dashed lines); 0.75 (dash-dotted lines); 1 (dotted lines); and the following $\alpha$ values, as well: $\hat{\alpha} \equiv 8.3 \times 10^{-8} \mathrm{~cm}^{2} / \mathrm{h}$ (black lines); $2 \times \hat{\alpha}$ (dark gray lines); $3 \times \hat{\alpha}$ (gray lines); and $4 \times \hat{\alpha}$ (light gray lines).

line; (b) dashed line; and (c) solid line. A significant increment is observed in all the cases reported here. Figure 2(b) shows the respective ratios between $j_{\min }$ and $j_{\min , 0}$ (black); and $j_{\max }$ and $j_{\max , 0}$ (gray); and Fig. 2 (c) is an equivalent plot, but considering the horizontal direction. This figure indicates that the presence of acid production clearly increases the cancer cell migration toward the blood vessel [Fig. 2(b)], implying an increased risk of metastasis. Furthermore, tumor becomes narrower in the presence of acid production [Fig. 2(c)].

As we have expressed above, the gap width is not uniform, and, due to the tumor sprouting in the upper region, it would not be convenient to define an average gap width considering the whole boundary. Our interest is focused on the lowest area of growing, because this is related with the risk of having metastasis. Furthermore, the mainly difference between tumor progressions with and without considering acid production is the front of propagation toward the nutrient source. In order to define an average gap width, denoted by $\Delta$, we just consider the nodes with $i \in\left[i_{c m}-a, i_{c m}+a\right]$, where $i_{c m}$ is the horizontal position of the tumor center of mass, and $a=\left(i_{\max }-i_{\min }\right) \times 0.1$. For those nodes, the vertical distance from the last node with at least one cancer cell to the first node with at least one healthy cell is taken; and $\Delta$ is defined as the average of those distances. Figure 3 shows $\Delta$ versus the minimal distance from the tumor to the vessel, $\left(j_{\min }\right)$, for different realizations. Figure 3 shows clearly three groups, the first one has the lowest maximum and it corresponds to $\alpha_{W}=5 \times 10^{-6} \mathrm{~cm}^{2} / \mathrm{s}, \gamma_{W}=0.7$ [as in Fig. 1(a)]; the curves in the middle represent the second one, which corresponds to $\alpha_{W}=5 \times 10^{-6} \mathrm{~cm}^{2} / \mathrm{s}, \gamma_{W}=0.9$ [as in Fig. 1(b)]; and the third group, corresponding to the highest maximum, includes realizations with $\alpha_{W}=2 \times 10^{-6} \mathrm{~cm}^{2} / \mathrm{s}, \gamma_{W}=0.7$ [as in Fig. 1(c)]. For all these groups there are realizations considering the following $p_{f}$ values: 0.25 (solid lines); 0.5 (dashed lines); 0.75 (dash-dotted lines); 1 (dotted lines); and $\alpha=\hat{\alpha} \equiv$ $8.3 \times 10^{-8} \mathrm{~cm}^{2} / \mathrm{h}$ (black lines); $2 \times \hat{\alpha}$ (dark gray lines); $3 \times$ $\hat{\alpha}$ (gray lines); and $4 \times \hat{\alpha}$ (light gray lines). Parameters $p_{f}$ and $\alpha$ were defined in rules R7 and R6, they represent the invasion probability and the cancer cell migration coefficient, respectively. Although experimental data are in correlation with the first group, the study of the second and third groups becomes interesting because the effects, that are already present in the first group, are amplified. Variations with $P_{D}$ were not included because they were not relevant $\left(P_{D}\right.$ was defined in rule R6, cancer cell migration takes place if the local amount of free nutrient is less than $P_{D}$ ). When tumors start growing there is no gap. Once cancer cells consume nutrients, the level of $\mathrm{H}^{+}$ion concentration increases and healthy cells start dying. The onset of gap increases the nutrient availability, because dead healthy cells do not consume; this also increases cancer cell consumption and thus $W$ local concentration. This positive feedback increases nutrient flow from the vessel to the tumor and contributes to the gap growing. However, after a while, nutrient availability is not enough to maintain all the new cancer cells, thus cancer cell migration become more active and the gap size starts decreasing. Once the active front is near to the vessel, the absorbent condition becomes more relevant and local $W$ concentration is reduced due to the loss of the $\mathrm{H}^{+}$ion through the vessel. When cancer cells arrive to the blood vessel, gap size turns zero by definition. Figure 3 shows that, although the gap size depends on $p_{f}$ and $\alpha$, the main influence in the gap evolution is given by acid production and diffusion. In general, for each group, the larger the cell migration coefficient, $\alpha$, the smaller the gap. On the other hand, the lower the healthy cell resistance, the larger the gap.

\section{CONCLUSIONS}

In this work we used a mathematical model to describe the formation and evolution of a gap between cancer and healthy cells due to an excess of $\mathrm{H}^{+}$ion concentration. Our model is based on space and nutrient competitions and considers a nonuniform distribution of nutrients. The gap onsets in the region with more consumption by cancer cells, depending on tumor activity, as well as, acid production and diffusion properties, the gap could surround the whole tumor-host interface. Our results suggest that although the gap itself is weakly correlated with intrinsic cancer cell migration properties and healthy cell resistance, its presence indicates an increased cancer cell mobility toward the regions with more nutrient availability.

In our model all cancer cells have the same migration coefficient. However, their effective mobility depends on the local conditions. Nutrient availability triggers reproduction; and thus, more consumption. The increase in nutrient consumption produces more acid; as a consequence, healthy cells die generating more space and nutrient availability for cancer cells. This feedback increases nutrient flow toward tumors and the activity of already active regions of tumors inducing cancer cell migration in those sectors. The combination of 
these key factors drives tumor evolution. Comparing these results with those for tumor evolutions without considering the excess of $\mathrm{H}^{+}$ions on the tissue, we can state that acid production increases the migration bias toward the nutrient source and thus, also increases the probability to invade by metastasis.

In this work, we consider that the network bottom line represents a blood vessel. With this scenario, the closer to the bottom, the more aggressive the tumor in the sense of metastasis. Our results indicate that acid production is associated with a faster propagation of the lowest front. If we analyze the minimal distance from the tumor to the vessel as a function of time (after a few weeks of seeding), we find that is linear, indicating a constant growth velocity (non-shown results). However, the gap size is not constant as a function of time. Even more, it is easy to find similar growth velocity for very different gap size evolutions. Most of the models, that consider acid-mediated tumor invasion, obtain a constant gap size for a given tumor dynamics, and according to this, correlation between growing speed can be analyzed. The main difference with our findings is that gap size is not constant and, for a given tumor dynamics, it depends on the distance to the nutrient source location. In particular, our model suggests that there is no strong correlation between the gap size and the tumor growth velocity as in Ref. [4], but it mainly depends on the distance to the nutrient source and the acid production. In this sense, it would be difficult to obtain information about growing speed from gap images. However, the presence of a gap can be consider a hallmark, because it indicates tumors with a high risk of metastasis; and if the gap is present in just a part of the tumor-host interface, this region should be associated with the more active tumor area, and it would also indicates the direction of growing.

\section{ACKNOWLEDGMENTS}

This work was partially supported by SeCyT-UNC and CONICET (PIP 11220150100644). S.A.M. thanks the hospitality of Prof. H. J. (Bert) Kappen at the Donders Institute for Neuroscience, Radboud University, Nijmegen, where this work was finished.
[1] O. Warburg, Science 123, 309 (1956).

[2] T. Han, D. Kang, D. Ji, X. Wang, W. Zhan, M. Fu, H. Xin, and J. Wang, Cell Adhes. Migr. 7, 395 (2013).

[3] Y. Kato, S. Ozawa, C. Miyamoto, Y. Maehata, A. Suzuki, T. Maeda, and Y. Baba, Cancer Cell Int. 13, 89 (2013).

[4] R. A. Gatenby and E. T. Gawlinski, Cancer Res. 56, 5745 (1996).

[5] R. A. Gatenby and E. T. Gawlinski, Cancer Res. 63, 3847 (2003).

[6] N. Martin, E. Gaffney, R. Gatenby, and P. Maini, J. Theor. Biol. 267, 461 (2010).

[7] J. McGillen, E. Gaffney, N. Martin, and P. Maini, J. Math. Biol. 68, 1199 (2014).

[8] P. Tracqui, Rep. Prog. Phys. 72, 056701 (2009).

[9] S. C. Ferreira, Jr., M. L. Martins, and M. J. Vilela, Phys. Rev. E 65, 021907 (2002).

[10] P. Macklin and J. Lowengrub, J. Theor. Biol. 245, 677 (2007).

[11] T. Risler and M. Basan, New J. Phys. 15, 065011 (2013).

[12] S. A. Menchón and C. A. Condat, Phys. Rev. E 78, 022901 (2008).

[13] M. Scalerandi, A. Romano, G. P. Pescarmona, P. P. Delsanto, and C. A. Condat, Phys. Rev. E 59, 2206 (1999).
[14] J. J. Casciari, S. V. Sotirchos, and R. M. Sutherland, J. Cell. Physiol 151, 386 (1992).

[15] A. Hochreiter-Hufford and K. S. Ravichandran, Cold Spring Harb. Perspect. Biol. 5, a008748 (2013).

[16] Y. Jiang, J. Pjesivac-Grbovic, C. Cantrell, and J. P. Freyer, Biophys. J. 89, 3884 (2005).

[17] P. Schornack and R. Gillies, Neoplasia 5, 135 (2003).

[18] G. R. Martin and R. K. Jain, Cancer Res. 54, 5670 (1994).

[19] J. Fang, M. Sullivan, and T. F. McCutchan, J. Biol. Chem. 279, 720 (2004).

[20] J. J. Casciari, S. V. Sotirchos, and R. M. Sutherland, Cancer Res. 48, 3905 (1988).

[21] D. Drasdo and S. Höhme, Phys. Biol. 2, 133 (2005).

[22] J. P. Freyer and R. M. Sutherland, J. Cell Physiol. 124, 516 (1985).

[23] A. C. Kole, B. E. C. Plaat, H. J. Hoekstra, W. Vaalburg, and W. M. Molenaar, J. Nucl. Med. 40, 381 (1999).

[24] M. Chaplain and A. Matzavinos, Mathematical modeling of spatio-temporal phenomena in tumor immunology, Tutorials in Mathematical Biosciences III: Cell Cycle, Proliferation, and Cancer (Springer, New York, 2006), pp. 131-186.

[25] K. R. Swanson, C. Bridge, J. D. Murray, and J. E. C. Alvord, J. Neurol. Sci. 216, 1 (2003). 